\title{
A Piecewise Type-2 Fuzzy Regression Model
}

\author{
Narges Shafaei Bajestani ${ }^{1}$, Ali Vahidian Kamyad ${ }^{2 *}$, Assef Zare ${ }^{3}$ \\ ${ }^{1}$ Department of Electrical Engineering, Science and Research Branch, Islamic Azad University, Tehran, Iran \\ E-mail: Narges.shafaei@gmail.com
}

${ }^{2}$ Department of Electrical Engineering, Science and Research Branch, Islamic Azad University, Tehran, Iran E-mail: a.vahidian.kamyad@gmail.com

${ }^{3}$ Department of Electrical Engineering, Gonabad Branch, Islamic Azad University, Gonabad, Iran E-mail: assefzare@gmail.com

Received 22 February 2016

Accepted 7 February 2017

\begin{abstract}
The type-2 fuzzy logic system permits us to model uncertainties existing in membership functions. Accordingly, this study aims to propose a linear and a piecewise framework for an interval type-2 fuzzy regression model based on the existing possibilistic models. In this model, vagueness is minimized, under the circumstances where the $\mathrm{h}$ cut of observed value is included in predicted value. In this model both primary and secondary membership function of predicted value fit the observed value. Developing the proposed model to piecewise model makes it helpful in dealing with the fluctuating data. This model, without the additional complexities, demonstrates its ability compared to previous type-2 fuzzy models.
\end{abstract}

Keywords: Interval type-2 fuzzy regression, Quadratic programming, type-2 fuzzy piecewise regression.

\section{Introduction}

Statistical linear regression has long been used in almost every field of science. Developed by H. Tanaka et al. ${ }^{1-}$ 3 , the fuzzy regression models have been applied to modeling systems involving vague phenomena. Fuzzy regression techniques can generally be classified into two distinct categories. The first category, suggested by P. Diamond ${ }^{4}$, is an adaptation of the classical least squares method. The second category, known as possibilistic regression ${ }^{1-3}$, reduces the problem of obtaining fuzzy coefficients of a regression model to one mathematical programming.

The fuzzy regression analysis can be applied to many real-life issues ${ }^{5-8}$. In which the strict supposition of classical regression models cannot be satisfied. For example, people infrequently use numerical expressions to judge things in their life. The identification, the judgment and evaluation that peoples actually perform are usually expressed in qualitative linguistic terms that can be modeled with fuzzy sets. Many researchers have worked in the field of fuzzy linear regression (FLR) ${ }^{9-13}$. However, to this date, most models of fuzzy regression analysis have focused only on type-1 fuzzy sets, while the type-2 fuzzy logic systems (T2FLS) can help deal with even higher levels of uncertainty in real world applications. Type- 2 fuzzy rules are more complicated than type-1 fuzzy rules, because they use of type-2 fuzzy sets in antecedent or consequent parts. Therefore, most of the type-2 FLS studies only concern with interval type-2 FLSs (IT2FLS).

\footnotetext{
* Corresponding author, Address: Department of Electrical Engineering, Science and Research Branch, Islamic Azad University, Tehran, Iran. Email: a.vahidian.kamyad@gmail.com
} 
Some researchers have used type-2 fuzzy regressions as the modeling structure. Y. Wei, J. Watada have been built a type-2 Fuzzy Qualitative Regression Model ${ }^{15}$, which substantially is a simpler version of our model when $\mathrm{H}=0$. In their article, Wei and Watada imply that they have used a general type-2 fuzzy number while it seems that they have used interval type2. O. Poleshchuk, E. Komarov present a regression model for interval type-2 fuzzy sets based on the least squares estimation technique, but there isn't an example to guarantee the efficient performance of their proposed approach ${ }^{16}$. E. Hosseinzadeh et al present a weighted goal programming approach to fuzzy linear regression with crisp inputs and type-2 fuzzy outputs ${ }^{17}$. But this model only has tried to close the membership functions of observed and estimated responses by closing some of their parameters. Shafaei Bajestani et al used a new interval type-2 fuzzy regression model to forecast Taiwan stock index ${ }^{18,19}$. It seems that none of the studies mentioned above could well model the type- 2 fuzzy regression; instead they have reduced their model to only some points of type-2 fuzzy numbers. Also, the piecewise model than can model the large variation data has not been discussed in these studies.

This study aims to propose a framework for an interval type-2 possibilistic fuzzy regression model. Since interval type-2 fuzzy membership functions could be represented only by its Footprint of Uncertainty (FOU). This model has been built based on the possibility model for upper membership function (UMF) and necessity model for lower membership function (LMF). Moreover, the next section introduces interval type-2 fuzzy piecewise regression (IT2FPR) model's construction, which hasn't been discussed in the existing literature.

The rest of this paper is organized as follows: Section 2 reviews the relevant literature mentioned in this study; Section 3 describes the proposed type-2 fuzzy regression; Section 4 demonstrates and compares the ability of the proposed models in two examples; Finally, Section 5 is the conclusion and implications.

\section{Review}

In this section, the concepts and definitions used in this research are reviewed.

Definition 1. (Type-2 fuzzy sets) A T2FS is denoted by

$$
\tilde{A}=\int_{\forall x \in X} \int_{\forall u \in J_{x} \subseteq[0,1]} \mu_{\tilde{A}}(x, u) /(x, u)
$$

where $\iint$ denotes the union over all admissible domain values $\mathrm{x}$ and secondary domain values $\mathrm{u}$, and $\mu_{\tilde{A}}(x, u)$ is a type-2 membership function. A T2FS is three-

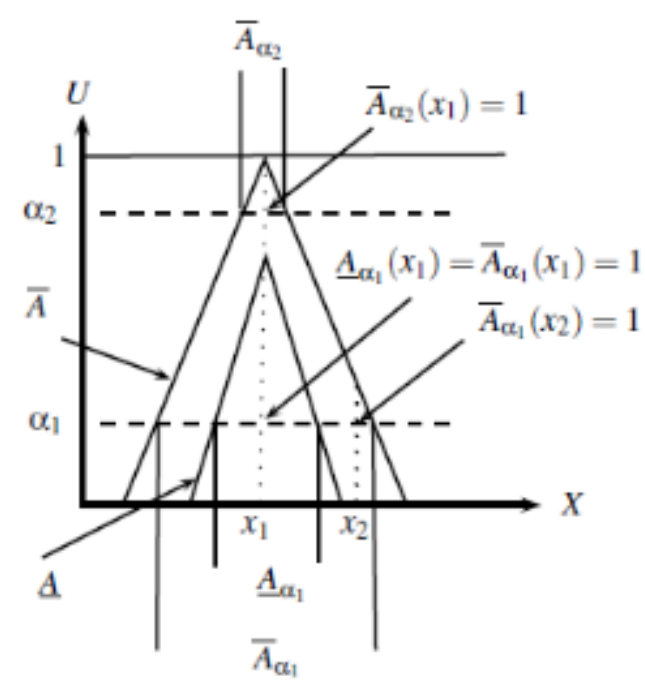

Fig. 1. $\alpha$-cuts of IVFS ${ }^{14}$

dimensional. The Vertical Slice (VS) in the twodimensional plane of the $\mathrm{u}$ and $\mu_{\tilde{A}}(x, u)$ axes for a special value of $x$, say $x^{\prime}$, is defined as follows:

$$
V S\left(x^{\prime}\right)=\mu_{\tilde{A}}\left(x^{\prime}, u\right)=\mu_{\tilde{A}}\left(x^{\prime}\right)=\int_{u \in J_{x^{\prime}}} f_{x^{\prime}}(u) / u
$$

Where $f_{x^{\prime}}(u) \in[0,1]$ is called the secondary grade and $J_{x}$ represent the domain of the secondary membership function called secondary domain. The VS is a type-1 fuzzy set (T1FS) in $[0,1]$.

The FOU is derived from the union of all primary memberships:

$$
\operatorname{FOU}(\tilde{A})=\int_{x \in X} J_{x}
$$

The FOU is bounded by two membership function, a lower $\underline{\mu_{\tilde{A}}(x)}$ and an upper $\overline{\mu_{\tilde{A}}(x)}$. The FOU can be described in terms of its lower and upper membership functions (LMF and UMF) and which themselves are T1FS:

$$
\operatorname{FOU}(\tilde{A})=\int_{x \in X}\left[\underline{\left.\mu_{\tilde{A}}(x), \overline{\mu_{\tilde{A}}(x)}\right]}\right.
$$

Definition 2. (Interval type-2 fuzzy sets) An interval type-2 fuzzy set (IT2FS) is defined as a T2FS whose all secondary grades are of unity, i.e. An IT2FS can be completely determined using its FOU.

Definition 3. (Normal T1FS) A T1FS say $\tilde{A}$, is said to be normal is its height is equal to 1 , i.e. $\sup \mu_{\tilde{A}}(x)=1$. 
Definition 4. (Normal IT2FS) An IT2FS $\tilde{A}$, is said to be normal if its upper membership function (MF) is normal, i.e. $\sup \overline{\mu_{\tilde{A}}(x)}=1$

Definition 5. (Perfectly Normal IT2FS) An IT2FS say $\tilde{A}$, is said to be perfectly normal if both its upper and lower MFs are normal, i.e. $\sup \overline{\mu_{\tilde{A}}(x)}=\sup \mu_{\tilde{A}}(x)=1$

Definition 6. Let $\tilde{A}=\left\langle c ; \alpha_{1}, \beta_{1} ; \alpha_{2}, \beta_{2}\right\rangle$, $\tilde{B}=\left\langle c^{\prime} ; \alpha_{1}^{\prime}, \beta_{1}^{\prime} ; \alpha_{2}^{\prime}, \beta_{2}^{\prime}\right\rangle$ are IT2FN and $w_{i}>0$ for $\mathrm{i}=1,2, \ldots, 5$, define $d_{w}$ as follow ${ }^{17}$ :

$$
\begin{aligned}
& d_{w}(\tilde{A}, \tilde{B})=w_{1}\left|c-c^{\prime}\right|+w_{2}\left|\alpha_{1}-\alpha_{1}^{\prime}\right| \\
& \quad+w_{3}\left|\beta_{1}-\beta_{1}^{\prime}\right|+w_{4}\left|\alpha_{2}-\alpha_{2}^{\prime}\right|+w_{5}\left|\beta_{2}-\beta_{2}^{\prime}\right|
\end{aligned}
$$

Definition 7. Let $\left(\underline{A}_{\propto}, \bar{A}_{\propto}\right)$ be a short notation for taking the $\alpha$-cuts of the LMF, $\underline{A}$, UMF, $\bar{A}$, of an IT2FS, $A^{V}$. Then $\left[\mu_{A_{x}}(x), \mu_{\bar{A}_{x}}(x)\right]$ and $\left[\mu_{\propto \propto A_{x}}(x), \mu_{\propto \bar{A}_{x}}(x)\right]$ are closed intervals across all domain value $x$.

Definition 8. (IT2FS $\alpha$-cuts) The $\alpha$-cuts of an IT2FS, $A^{V}$ is a non-fuzzy set defined as follows: $A_{\alpha}^{V}\left(\underline{A}_{\propto}, \bar{A}_{\propto}\right)$ where $\mu_{A_{\alpha}^{V}}(x)=\left[\mu_{A_{x}}(x), \mu_{\bar{A}_{x}}(x)\right]$ (Fig. 1$)^{14}$. We assume that a fuzzy phenomenon can be presented as a fuzzy system of equations which, in turn, can be described by the fuzzy function $\hat{Y}_{i}=A_{1} x_{i 1}+A_{2} x_{i 2}+\ldots+A_{q} x_{i q}$ in which $A_{j}$ is the fuzzy sets with triangular membership functions $\left(a_{j}, b_{j}, c_{j}\right)$ , $\mathrm{i}=1,2, \ldots, n$. Therefore the membership function of the fuzzy linear function $\hat{Y}_{i}$ is obtained as the triangular membership function $\left(C_{i 1}, C_{i 3}, C_{i 2}\right)$ in which:

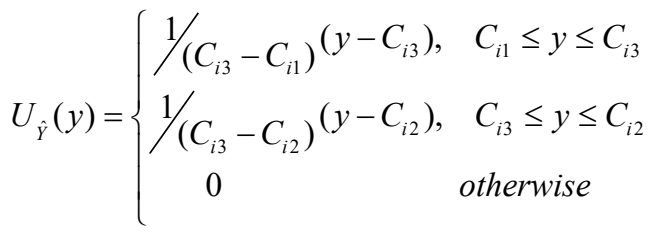

$$
\begin{aligned}
& C_{i 1}=\sum_{j=1}^{q} a_{j} x_{i j}, \quad C_{i 2}=\sum_{j=1}^{q} c_{j} x_{i j}, \quad C_{i 3}=\sum_{j=1}^{q} b_{j} x_{i j}
\end{aligned}
$$

The membership function of the observed output data $Y_{i}$ also should have the same special form $\left(p_{i}, q_{i}, r_{i}\right)$. According to the possibility and nessesity models of tanaka, the type-1 fuzzy regression model can be rewiten as :

$$
\begin{aligned}
& Y_{i} \subseteq_{h} \overline{\hat{Y}}_{i}=\bar{A}_{1} x_{i 1}+\bar{A}_{2} x_{i 2}+\ldots+\bar{A}_{q} x_{i q} \\
& Y_{i} \supseteq_{h} \underline{\hat{Y}}=\underline{A}_{1} x_{i 1}+\underline{A}_{2} x_{i 2}+\ldots+\underline{A}_{q} x_{i q}
\end{aligned}
$$

where $\overline{\hat{Y}}_{i}$ and $\hat{Y}_{i}$ are upper and lower estimation respectively and $\bar{A}_{j}=\left(\bar{a}_{i}, \overline{\mathrm{b}}_{j}, \bar{c}_{j}\right), \underline{A}_{j}=\left(\underline{a}_{i}, \underline{\mathrm{b}}_{j}, \underline{c}_{j}\right)$ are the coeficient of their model.

$Y_{i} \subseteq_{h} \overline{\hat{Y}}_{i}$ shows that h-cut of the upper estimation $\overline{\hat{Y}}_{i}$ always includes h-cut of the observed value $Y_{i}$. $Y_{i} \supseteq_{h} \underline{\underline{Y}}_{i}$ shows that h-cut of the observed value $Y_{i}$ always includes h-cut of the lower estimation $\underline{\underline{Y}}_{i}$.

The objective function is the sum of squared spreads of the upper and lower estimations.

The type-1 fuzzy regression problem can be reduced to the following two quadratic programming $(\mathrm{QP})^{2,20}$. H is chosen as the degree of the fitting of the fuzzy linear model by the decision-maker.

Possibility model:

$$
\begin{aligned}
& \min \sum_{i=1}^{n} \sum_{j=1}^{q}\left(\left(\bar{c}_{j}-\bar{a}_{j}\right) x_{i j}\right)^{2} \\
& \text { s.t } \\
& \bar{C}_{i 3}-(1-H)\left(\bar{C}_{i 3}-\bar{C}_{i 1}\right) \leq q_{i}-(1-H)\left(q_{i}-p_{i}\right) \\
& \bar{C}_{i 3}+(1-H)\left(\bar{C}_{i 2}-\bar{C}_{i 3}\right) \geq q_{i}+(1-H)\left(r_{i}-q_{i}\right)
\end{aligned}
$$

Necessity model:

$$
\begin{aligned}
& \max \sum_{i=1}^{n} \sum_{j=1}^{q}\left(\left(\underline{c}_{j}-\underline{a}_{j}\right) x_{i j}\right)^{2} \\
& \text { s.t } \\
& \underline{C}_{i 3}-(1-H)\left(\underline{C}_{i 3}-\underline{C}_{i 1}\right) \geq q_{i}-(1-H)\left(q_{i}-p_{i}\right) \\
& \underline{C}_{i 3}+(1-H)\left(\underline{C}_{i 2}-\underline{C}_{i 3}\right) \leq q_{i}+(1-H)\left(r_{i}-q_{i}\right)
\end{aligned}
$$

\section{Proposed type-2 fuzzy regression}

Type-1 FLSs cannot fully handle high levels of uncertainties existing in most of the real world applications. The reason lies behind the fact that type-1 FLSs employs crisp and "precise" type-1 fuzzy sets. A type-2 FLS can handle higher uncertainty levels to produce improved performance. Type- 2 FLSs have a variety of real world applications, in business and finance domains, in electrical energy domain, real-world automatic control, healthcare and medical domains ${ }^{21-24}$. The membership functions of type- 2 fuzzy sets are three dimensional and include a footprint of uncertainty. It is 
the third dimension of type-2 fuzzy sets, i.e. the footprint of uncertainty that additional degrees of freedom are provided, which in turn makes it possible to model and handle uncertainties ${ }^{25}$.

Interval type- 2 fuzzy sets are the most widely used type-2 fuzzy sets, since on the one hand they are simple to use and, on the other hand it is very difficult to justify the use of any kind of type- 2 fuzzy set. In this case, the membership function is an interval type-2 fuzzy set which can be represented only by its FOU.

In this section, at first, proposed interval type-2 fuzzy regression model was formulated. Thereafter, this model developed to piecewise type-2 fuzzy regression and multivariate model.

Concerning Arianna Mencattini et al. ${ }^{26}$, we will denote this kind of type-2 interval as $\tilde{A}_{j}=\left[\left[a_{u j} a_{l j}\right], b_{j},\left[c_{l j} c_{u j}\right] \quad\right.$ such that $L M F_{\tilde{A}}=\left(a_{l j}, b_{j}, c_{l j}\right), \quad U M F_{\tilde{A}}=\left(a_{u j}, b_{j}, c_{u j}\right) \quad$ and $a_{u j} \leq a_{l j} \leq b_{j} \leq c_{l j} \leq a_{u j}$.

The observed output data is the interval type-2 fuzzy number $\tilde{y}_{i}=\left(\left[p_{i u} p_{i l}\right], q_{i},\left[r_{i l} r_{i u}\right]\right)$.

$\tilde{Y}_{i}=\left(\tilde{Y}_{i}, \overline{\tilde{Y}}_{i}\right)$ is the predicted output and $\tilde{y}_{i}=\left(\underline{\tilde{y}}_{i}, \overline{\tilde{y}}_{i}\right)$ is the observed output. The basic idea of our approach based on Tanaka's approach is to minimize the fuzziness of the model by minimizing the total spread of the fuzzy coefficients, subject to including all the given data. In type-2 fuzzy regression these should be satisfied for primary membership functions and secondary membership functions.

\subsection{Proposed linear interval type-2 fuzzy regression}

Since an IT2FS can be completely determined using its FOU and FOU is bounded by two membership functions LMF and UMF (I.e.by two type-1 MFs), this model has been built based on the possibility model for UMF and the necessity model for LMF. These two models, besides minimization the vagueness in secondary membership function, help to find an appropriate fit for the predicted value into the observed value.

To formulate a fuzzy linear regression model, the followings are assumed to hold.

1. The data can be represented by an interval type-2 fuzzy linear model,

$\tilde{Y}_{i}=\tilde{A}_{1} x_{i 1}+\tilde{A}_{2} x_{i 2}+\ldots+\tilde{A}_{q} x_{i q}$

where $\tilde{Y}_{i}$ and $\tilde{A}_{j}$ are perfectly normal IT2FS and $x_{j}$ is positive.
According to the model, the supports of LMF and UMF were obtained as the following:

$$
\begin{aligned}
& \tilde{\mathrm{Y}}_{i}=\left(\left[C_{i 1 U}, C_{i 1 L}\right], C_{i 3},\left[C_{i 2 L}, C_{i 2 U}\right]\right)= \\
& \left.\qquad\left[a_{i u 1}, a_{i l 1}\right], b_{i 1},\left[c_{i l 1}, a_{i u 1}\right]\right] x_{i 1}+\ldots \\
& +\left[\left[a_{i u n}, a_{\mathrm{ilq}}\right], b_{q},\left[c_{\mathrm{ilq}}, a_{i u q}\right]\right] x_{i q} \\
& =\left[\left[a_{i u 1} x_{i 1}, a_{i l 1} x_{i 1}\right], b_{i 1} x_{i 1},\left[c_{i l 1} x_{i 1}, a_{i u 1} x_{i 1}\right]\right]+\ldots \\
& +\left[\left[a_{i u n} x_{i q}, a_{\mathrm{ilq}} x_{i q}\right], b_{i q} x_{i q},\left[c_{i 1 \mathrm{q}} x_{i q}, a_{i u q} x_{i q}\right]\right]
\end{aligned}
$$

Thus:

$$
\begin{array}{ll}
C_{i 1 U}=\sum_{j=1}^{q} a_{u j} x_{i j}, & C_{i 1 L}=\sum_{j=1}^{q} a_{l j} x_{i j}, \quad C_{i 3}=\sum_{j=1}^{q} b_{j} x_{i j} \\
C_{i 2 L}=\sum_{j=1}^{q} c_{l j} x_{i j}, & C_{i 2 U}=\sum_{j=1}^{q} c_{i j} x_{i j}
\end{array}
$$

2. The degree of the fitting of the estimated value $\tilde{Y}_{i}=\left(\underline{\tilde{Y}}_{i}, \overline{\tilde{Y}}_{i}\right)=\left(\left[C_{i 1 U}, C_{i 1 L}\right], C_{i 3},\left[C_{i 2 L}, C_{i 2 U}\right]\right) \quad$ to observed value $\tilde{y}_{i}=\left(\underline{\tilde{y}}_{i}, \overline{\tilde{y}}_{i}\right)=\left(\left[p_{i u}, p_{i l}\right], q_{i},\left[r_{i l}, r_{i u}\right]\right)$ is measured by the h-level sets of the observed and the predicted fuzzy numbers respectively, where h-cut have defined based on definition 8 .

In fact, the fundamental idea is to obtain parameters $a_{u j}, a_{l j}, b_{j}, c_{l j}, c_{u j}$ of coefficient $\tilde{A}_{j}$ such that:

$$
\underline{\tilde{Y}}_{i} \subset_{h} \underline{\tilde{y}}_{i} \quad, \quad \overline{\tilde{y}}_{i} \subset_{h} \overline{\tilde{Y}}_{i}
$$

It means that the possibility model for UMFs and the necessity model for LMFs are used respectively. Because it is enough to close the membership functions of the observed and predicted values as much as possible and an h-cut of the observed value is included in the predicted value (Fig.2).

3. The objective function is defined by $J=J_{1}+J_{2}-J_{3}+J_{4}$.

$$
\begin{aligned}
& J_{1}=\sum_{i=1}^{n} \sum_{j=1}^{q}\left(\left(a_{u j}-a_{l j}\right) x_{i j}\right)^{2}+\sum_{i=1}^{n}\left(\left(c_{u j}-c_{l j}\right) x_{i j}\right)^{2} \\
& J_{2}=\sum_{i=1}^{n} \sum_{j=1}^{q}\left(\left(c_{u j}-a_{u j}\right) x_{i j}\right)^{2} \\
& J_{3}=\sum_{i=1}^{n} \sum_{j=1}^{q}\left(\left(c_{l j}-a_{l j}\right) x_{i j}\right)^{2} \\
& J_{4}=\sum_{i=1}^{n}\left(\mathrm{q}_{i}-\sum_{j=1}^{n} b_{j} x_{i j}\right)^{2}
\end{aligned}
$$




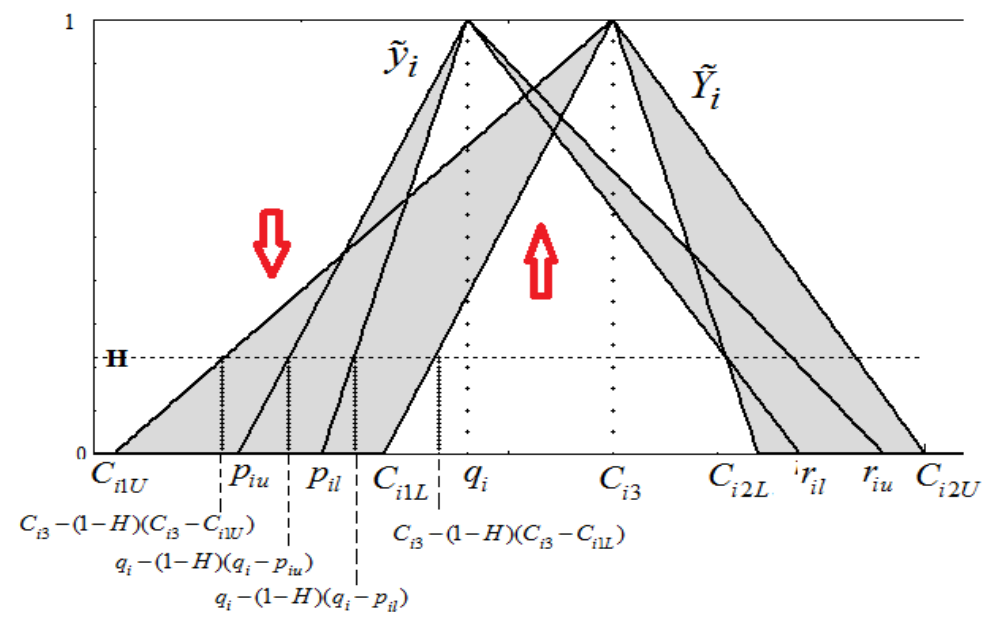

Fig. 2. Degree of fitting of $\tilde{\boldsymbol{Y}}$ to $\tilde{y}$

That minimizing of $J_{1}$ minimizes the vagueness of secondary membership function; and $J_{2}$ minimizes the vagueness of LMF of $\tilde{Y}$ that is a type-1 fuzzy set. $J_{4}$ minimizes the distance point with the highest membership value in a fuzzy estimated response and the point with the highest membership value in the corresponding observed response. But $J_{3}$ is used for the necessity problem, and hence it should be maximized.

More specially, our problem is to find out the fuzzy parameters $a_{u j}, a_{l j}, b_{j}, c_{l j}, c_{u j}$, which are the solution of the following two QP problems besides two extra objective functions (Fig.2):

$$
\begin{aligned}
& \min \mathrm{J}_{2} \\
& \text { s.t } \\
& C_{i 3}-(1-H)\left(C_{i 3}-C_{i 1 U}\right) \leq q_{i}-(1-H)\left(q_{i}-p_{i u}\right) \\
& C_{i 3}+(1-H)\left(C_{i 2 U}-C_{i 3}\right) \geq q_{i}+(1-H)\left(r_{i u}-q_{i}\right)
\end{aligned}
$$

$$
\begin{aligned}
& \max \mathrm{J}_{3} \\
& \text { s.t } \\
& C_{i 3}-(1-H)\left(C_{i 3}-C_{i 1 L}\right) \geq q_{i}-(1-H)\left(q_{i}-p_{i l}\right) \\
& C_{i 3}+(1-H)\left(C_{i 2 L}-C_{i 3}\right) \leq q_{i}-(1-H)\left(r_{i l}-q_{i}\right)
\end{aligned}
$$

where $\mathrm{H}$ is chosen as the degree of the fitting of the fuzzy linear model by the decision-maker.

QP problem of (11) and (12) are possibility and necessity model that used for UMF and LMF respectively.
More specifically, we can rewrite our problem as following:

$$
\begin{aligned}
& \text { Min } \quad J=J_{1}+J_{2}-J_{3}+J_{4} \\
& \text { s.t } \\
& C_{i 3}-(1-H)\left(C_{i 3}-C_{i 1 U}\right) \leq q_{i}-(1-H)\left(q_{i}-p_{i u}\right) \\
& C_{i 3}+(1-H)\left(C_{i 2 U}-C_{i 3}\right) \geq q_{i}+(1-H)\left(r_{i u}-q_{i}\right) \\
& C_{i 3}-(1-H)\left(C_{i 3}-C_{i 1 L}\right) \geq q_{i}-(1-H)\left(q_{i}-p_{i l}\right) \\
& C_{i 3}+(1-H)\left(C_{i 2 L}-C_{i 3}\right) \leq q_{i}-(1-H)\left(r_{i l}-q_{i}\right) \\
& a_{u j} \leq a_{l j} \leq b_{j} \leq c_{l j} \leq c_{u j}
\end{aligned}
$$

\subsection{Piecewise type-2 fuzzy regression}

Linear models are inadequate in handling fluctuating data. Therefore, it is necessary for the model to be developed to the piecewise model. One of the advantages of our proposed model is that it can be easily extended to the piecewise model. Two QP formulations are presented to determine the necessity area for LMF and the possibility area for UMF by the interval type-2 fuzzy piecewise regression model.

The data can be represented by a piecewise interval type-2 fuzzy linear model:

$$
\begin{aligned}
& \tilde{Y}_{i}=h\left(x_{i j}\right)+\sum_{t=1}^{k-1} \tilde{B}_{t}\left(\left|x_{i j}-P_{t}\right|+x_{i j}-P_{t}\right) / 2 \\
& h\left(x_{i j}\right)=\tilde{A}_{0}+\tilde{A}_{1} x_{i j}
\end{aligned}
$$

If $\mathrm{P}$ is a change-point, then

$\left(\left|x_{j}-P\right|+x_{j}-P\right) / 2=\left\{\begin{array}{c}x_{j}-P \quad \text { if } \quad x_{j}>P \\ 0 \text { if } \quad x_{j} \leq P\end{array}\right.$

$P=\left\{P_{1}, P_{2}, \ldots, P_{k}\right\}$ are the values of variable $\mathrm{x}$ subject to an ordering constraint $P_{1}<P_{2}<\ldots<P_{k}, k \leq n$. The 
practitioner can specify the number and position of change-points according to the data distribution.

In this model only calculation of $C_{i 1 U}, C_{i 1 L}, C_{i 3}, C_{i 2 L}, C_{i 2 U}$ are different from Linear model.

Preposition: If $\tilde{B}_{t}=\left(\left[b_{t u l} b_{t l l}\right], b_{t c},\left[\left[b_{t l r} b_{t u r}\right]\right.\right.$ :

$C_{i 1 U}=a_{u 0}+a_{u 1} x_{i j}+\sum_{t=1}^{k-1} b_{t u l}\left(\left|x_{i j}-P_{t}\right|+x_{i j}-P_{t}\right) / 2$

$C_{i 1 L}=a_{l 0}+a_{l 1} x_{i j}+\sum_{t=1}^{k-1} b_{t l l}\left(\left|x_{i j}-P_{t}\right|+x_{i j}-P_{t}\right) / 2$

$C_{i 3}=b_{0}+b_{1} x_{i j}+\sum_{t=1}^{k-1} b_{t c}\left(\left|x_{i j}-P_{t}\right|+x_{i j}-P_{t}\right) / 2$

$C_{i 2 L}=c_{l 0}+c_{l 1} x_{i j}+\sum_{t=1}^{k-1} b_{t l r}\left(\left|x_{i j}-P_{t}\right|+x_{i j}-P_{t}\right) / 2$

$C_{i 2 U}=c_{u 0}+c_{u 1} x_{i j}+\sum_{t=1}^{k-1} b_{t u r}\left(\left|x_{i j}-P_{t}\right|+x_{i j}-P_{t}\right) / 2$

Proof:

$$
\begin{aligned}
& h\left(x_{i j}\right)=\tilde{A}_{0}+\tilde{A}_{1} x_{i j} \\
& =\left[\left[a_{i u 0}, a_{i l 0}\right], b_{i 0},\left[c_{i l 0}, \mathrm{c}_{i u 0}\right]\right] \\
& \quad+\left[\left[a_{i u 1}, a_{i l 1}\right], b_{i 1},\left[c_{i l 1}, \mathrm{c}_{i u 1}\right]\right] x_{i j} \\
& =\left[\left[a_{i u 0}+a_{i u 1} x_{i j}, a_{i u 0}+a_{i l 1} x_{i j}\right],\right. \\
& \left.\quad b_{i 0}+b_{i 1} x_{i j},\left[c_{i l 0}+c_{i l 1} x_{i j}, \mathrm{c}_{i u 0}+a_{i u 1} x_{i j}\right]\right]
\end{aligned}
$$

and

$$
\begin{aligned}
& \sum_{j=1}^{q} \sum_{t=1}^{k_{i}-1} \tilde{B}_{j t}\left(\left|x_{i j}-P_{t}\right|+x_{i j}-P_{t}\right) / 2= \\
& \sum_{j=1}^{q} \sum_{t=1}^{k_{i}-1}\left(\left[b_{t u l} b_{t l l}\right], b_{t c},\left[\left[b_{t l r} b_{t u r}\right]\right)\left(\left|x_{i j}-P_{t}\right|+x_{i j}-P_{t}\right) / 2\right.
\end{aligned}
$$

After a simple Addition and multiplication, $C_{i 1 U}, C_{i 1 L}, C_{i 3}, C_{i 2 L}, C_{i 2 U}$ were calculated and model converts to the following model:

$$
\begin{aligned}
& \text { Min } \quad J=J_{1}+J_{2}-J_{3}+J_{4} \\
& \text { s.t } \\
& b_{0}+b_{1} x_{i j}+\sum_{t=1}^{k-1} b_{t c}\left(\left|x_{i j}-P_{t}\right|+x_{i j}-P_{t}\right) / 2 \\
& \quad-(1-H)\left(b_{0}+b_{1} x_{i j}+\sum_{t=1}^{k-1} b_{t c}\left(\left|x_{i j}-P_{t}\right|+x_{i j}-P_{t}\right) / 2\right. \\
& \left.\quad-\left(a_{u 0}+a_{u 1} x_{i j}+\sum_{t=1}^{k-1} b_{t u l}\left(\left|x_{i j}-P_{t}\right|+x_{i j}-P_{t}\right) / 2\right)\right) \\
& \quad \geq q_{i}-(1-H)\left(q_{i}-p_{i u}\right)
\end{aligned}
$$

$$
\begin{aligned}
& b_{0}+b_{1} x_{i j}+\sum_{t=1}^{k-1} b_{t c}\left(\left|x_{i j}-P_{t}\right|+x_{i j}-P_{t}\right) / 2 \\
& +(1-H)\left(c_{u 0}+c_{u 1} x_{i j}+\sum_{t=1}^{k-1} b_{t u r}\left(\left|x_{i j}-P_{t}\right|+x_{i j}-P_{t}\right) / 2\right. \\
& \left.-\left(b_{0}+b_{1} x_{i j}+\sum_{t=1}^{k-1} b_{t c}\left(\left|x_{i j}-P_{t}\right|+x_{i j}-P_{t}\right) / 2\right)\right) \\
& \geq q_{i}+(1-H)\left(r_{i u}-q_{i}\right) \\
& b_{0}+b_{1} x_{i j}+\sum_{t=1}^{k-1} b_{t c}\left(\left|x_{i j}-P_{t}\right|+x_{i j}-P_{t}\right) / 2 \\
& -(1-H)\left(b_{0}+b_{1} x_{i j}+\sum_{t=1}^{k-1} b_{t c}\left(\left|x_{i j}-P_{t}\right|+x_{i j}-P_{t}\right) / 2\right. \\
& \left.-\left(a_{l 0}+a_{l 1} x_{i j}+\sum_{t=1}^{k-1} b_{t l l}\left(\left|x_{i j}-P_{t}\right|+x_{i j}-P_{t}\right) / 2\right)\right) \\
& \geq q_{i}-(1-H)\left(q_{i}-p_{i l}\right) \\
& b_{0}+b_{1} x_{j}+\sum_{t=1}^{k-1} b_{t c}\left(\left|x_{i j}-P_{t}\right|+x_{i j}-P_{t}\right) / 2 \\
& +(1-H)\left(c_{l 0}+c_{l 1} x_{i j}+\sum_{t=1}^{k-1} b_{t l r}\left(\left|x_{i j}-P_{t}\right|+x_{i j}-P_{t}\right) / 2\right. \\
& \left.-\left(b_{0}+b_{1} x_{i j}+\sum_{t=1}^{k-1} b_{t c}\left(\left|x_{i j}-P_{t}\right|+x_{i j}-P_{t}\right) / 2\right)\right) \\
& \geq q_{i}-(1-H)\left(r_{i l}-q_{i}\right) \\
& a_{u j} \leq a_{l j} \leq b_{j} \leq c_{l j} \leq c_{u j}
\end{aligned}
$$

This can be rewritten like the linear model in simple form:

$$
\begin{aligned}
& \text { Min } \quad J=J_{1}+J_{2}-J_{3}+J_{4} \\
& \text { s.t } \\
& C_{i 3}-(1-H)\left(C_{i 3}-C_{i 1 U}\right) \leq q_{i}-(1-H)\left(q_{i}-p_{i u}\right) \\
& C_{i 3}+(1-H)\left(C_{i 2 U}-C_{i 3}\right) \geq q_{i}+(1-H)\left(r_{i u}-q_{i}\right) \\
& C_{i 3}-(1-H)\left(C_{i 3}-C_{i 1 L}\right) \geq q_{i}-(1-H)\left(q_{i}-p_{i l}\right) \\
& C_{i 3}+(1-H)\left(C_{i 2 L}-C_{i 3}\right) \leq q_{i}-(1-H)\left(r_{i l}-q_{i}\right) \\
& a_{u j} \leq a_{l j} \leq b_{j} \leq c_{l j} \leq c_{u j}
\end{aligned}
$$

\subsection{Multivariate piecewise type-2 fuzzy regression}

In many applications, there is more than one input, In this regard, to be able to deal with the problem of multiple inputs; multivariate model was required to be formulated.

Multivariate case can be viewed as a straightforward generalization of the previous case so that the used concepts are the same. 
The output may be generally related to the $\mathrm{q}$ input. Every input could have some change-point. The multivariate model is as follow:

$$
\begin{aligned}
& \tilde{Y}_{i}=h\left(x_{i j}\right)+\sum_{j=1}^{q} \sum_{t=1}^{k_{i}-1} \tilde{B}_{j t}\left(\left|x_{i j}-P_{t}\right|+x_{i j}-P_{t}\right) / 2 \\
& h\left(x_{i j}\right)=\tilde{A}_{0}+\sum_{j=1}^{q} \tilde{A}_{1} x_{i j}
\end{aligned}
$$

In the multivariate model $C_{i 1 U}, C_{i 1 L}, C_{i 3}, C_{i 2 L}, C_{i 2 U}$ are calculated as follow:

$$
\begin{aligned}
& C_{i 1 U}=a_{u 0}+\sum_{j=1}^{q} a_{u j} x_{i j}+\sum_{j=1}^{q} \sum_{t=1}^{k_{i}-1} b_{j t u l}\left(\left|x_{i j}-P_{t}\right|+x_{i j}-P_{t}\right) / 2 \\
& C_{i 1 L}=a_{t 0}+\sum_{j=1}^{q} a_{l j} x_{i j}+\sum_{j=1}^{q} \sum_{t=1}^{k_{i}-1} b_{j t l l}\left(\left|x_{i j}-P_{t}\right|+x_{i j}-P_{t}\right) / 2 \\
& C_{i 3}=b_{0}+\sum_{j=1}^{q} b_{j} x_{i j}+\sum_{j=1}^{q} \sum_{t=1}^{k_{i}-1} b_{j t c}\left(\left|x_{i j}-P_{t}\right|+x_{i j}-P_{t}\right) / 2 \\
& C_{i 2 L}=c_{l 0}+\sum_{j=1}^{q} c_{l j} x_{i j}+\sum_{j=1}^{q} \sum_{t=1}^{k_{i}-1} b_{j t l r}\left(\left|x_{i j}-P_{t}\right|+x_{i j}-P_{t}\right) / 2 \\
& C_{i 2 U}=c_{u 0}+\sum_{j=1}^{q} c_{u j} x_{j}+\sum_{j=1}^{q} \sum_{t=1}^{k_{i}-1} b_{j t u r}\left(\left|x_{i j}-P_{t}\right|+x_{i j}-P_{t}\right) / 2
\end{aligned}
$$

The QP problem in this case is the same in equation 17 with different values of $C_{i 1 U}, C_{i 1 L}, C_{i 3}, C_{i 2 L}, C_{i 2 U}$ calculated in Equation 20.

\section{Numerical results}

To evaluate the performance of the proposed model, a hybrid standard error of estimates (HS), standard deviation $\left(\mathrm{S}_{\tilde{y}}\right)$, hybrid correlation coefficient (HR), root mean square error (RMSE), and R- squared $\left(\mathrm{R}^{2}\right)$ were used and defined as follows ${ }^{16,17}$.

$$
\begin{aligned}
& S_{\tilde{y}}=\frac{\sum_{i=1}^{n} d_{w}\left(\tilde{y}_{i}, \overline{\tilde{y}}\right)}{n-1}, \quad \overline{\tilde{y}}=\frac{\sum_{i=1}^{n} \tilde{y}_{i}}{n} \\
& \mathrm{HR}^{2}=\frac{\sum_{i=1}^{n} d_{w}\left(\hat{\mathrm{y}}_{i}, \overline{\tilde{y}}\right)}{\sum_{i=1}^{n} d_{w}\left(\tilde{y}_{i}, \overline{\tilde{y}}\right)} \\
& H S=\frac{\sum_{i=1}^{n} d_{w}\left(\hat{\mathrm{y}}_{i}, \tilde{\mathrm{y}}_{i}\right)}{n-m-1} \\
& w_{1}=w_{2}=w_{3}=w_{4}=w_{5}=1, \mathrm{~m}=1
\end{aligned}
$$




\subsection{Example 2}

We use example 2 of Hosseinzadeh et al. paper for a multivariate model. The proposed model obtain the relationship between sodium percentage $\mathrm{SP}$, as a function of three soil variables including the percentage of organic matter content (OM), sand content (SAND) and SILT, as the independent variables ${ }^{17}$. $\mathrm{OM}=2$, $\mathrm{SAND}=24, \mathrm{SILT}=41,44$ are chosen as change-point, then:

$$
\begin{aligned}
\tilde{Y}_{i}= & ([2934.2], 34.2,[3559]) \\
+ & ([6.2711], 11.08,[11.4512 .38]) \mathrm{OM}_{j} \\
+ & ([-.0652-.0652],-.0652, \\
& {[-.0652-.0652]) \mathrm{SAND}_{i} } \\
& +([.18 .19], .19,[.19 .21]) \text { SILT }_{i}
\end{aligned}
$$

$$
\begin{aligned}
+ & ([-3.5-3.5],-3.5,[-3.5-3.5]) \\
& \left.\left(\left|O M_{i}-2\right|+O M_{i}-2\right) / 2\right) \\
+ & ([-.59-6],-6.5,[-6.9-7.1]) \\
& \left.\left(\left|S A N D_{i}-24\right|+S A N D_{i}-24\right) / 2\right) \\
+ & ([-.34-.34],-.35,[-.35-.35]) \\
& \left.\left(\left|S I L T_{i}-41\right|+S I L T_{i}-41\right) / 2\right) \\
+ & ([-.52-.52],-.52,[-.52-.52]) \\
& \left.\left(\left|S_{L} T_{i}-44\right|+\operatorname{SILT}_{i}-44\right) / 2\right)
\end{aligned}
$$

\begin{tabular}{|c|c|c|c|c|c|}
\hline & HS & $\mathrm{HR}^{2}$ & $\mathrm{~S}_{\tilde{y}}$ & RMSE & $\mathrm{R}^{2}$ \\
\hline Type-1 fuzzy regression ${ }^{2}$ & 10.78 & 0.701 & 15.21 & 4.8 & $69.4 \%$ \\
\hline Poleshchuk et al approach ${ }^{16}$ & 7.19 & 0.758 & 14.45 & 3.7 & $78.3 \%$ \\
\hline WGP approach $^{17}$ & 5.58 & 0.779 & 11.10 & 3.1 & $81.35 \%$ \\
\hline Proposed Linear Type-2 fuzzy regression model & 9 & 0.766 & 10.98 & 2.6 & $83.51 \%$ \\
\hline Proposed piecewise type-2 fuzzy regression model & 5.5 & 0.803 & 8.12 & 2.1 & $87.52 \%$ \\
\hline
\end{tabular}

As Fig. 5 and table 2 (Continued) shows, RMSE, HS and $\mathrm{S}_{\tilde{y}}$ of proposed model with IT2FS coefficients is less than those of Poleshchuk et al.'s model and WGP model. In addition, the piecewise model has the maximum value of $R^{2}$ and $\mathrm{HR}^{2}$. These values in Table 2 show that, the predictive ability of our model is better than the predictive ability of earlier T2FS models.

Table 1. Comparing HS and RMSE in example 1

\begin{tabular}{cccccc}
\hline & $\mathrm{HS}$ & $\mathrm{HR}^{2}$ & $\mathrm{~S}_{\tilde{y}}$ & $\mathrm{RMSE}$ & $\mathrm{R}^{2}$ \\
\hline Type-1 fuzzy regression & \\
Poleshchuk et al approach & & & & \\
& 5.2 & 0.61 & 7.12 & 2.95 & $59.8 \%$ \\
WGP approach $^{17}$ & 3.77 & 0.68 & 6.20 & 1.82 & $66.2 \%$ \\
Proposed Linear Type-2 fuzzy regression model & 4.01 & 0.758 & 4.51 & 1.2 & $71.1 \%$ \\
Proposed piecewise type-2 fuzzy regression model & 1.90 & 1.08 & 4.12 & 0.11 & 99.8 \\
\hline
\end{tabular}

Table 2. Comparing HS and RME in multivariate model 


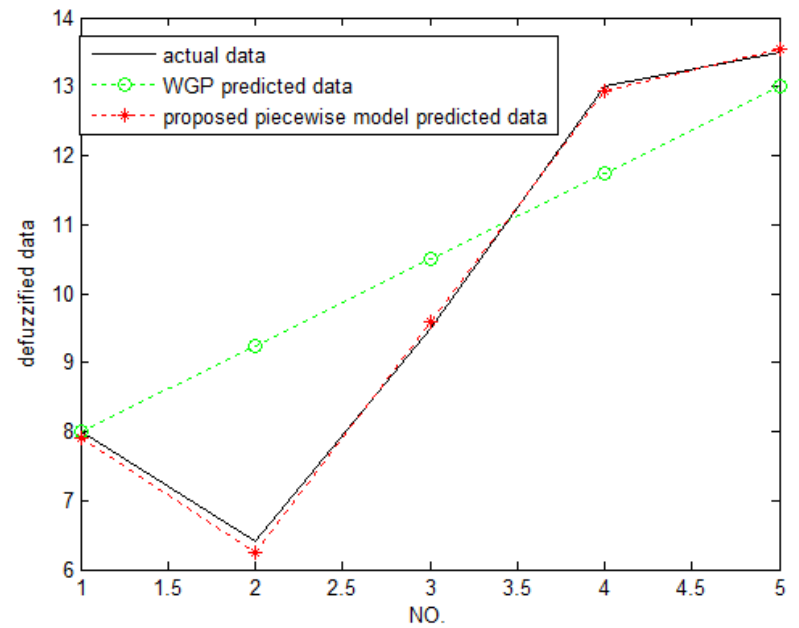

Fig. 3. Defuzzyfied actual values, WGP predicted values and proposed T2FLR model predicted values
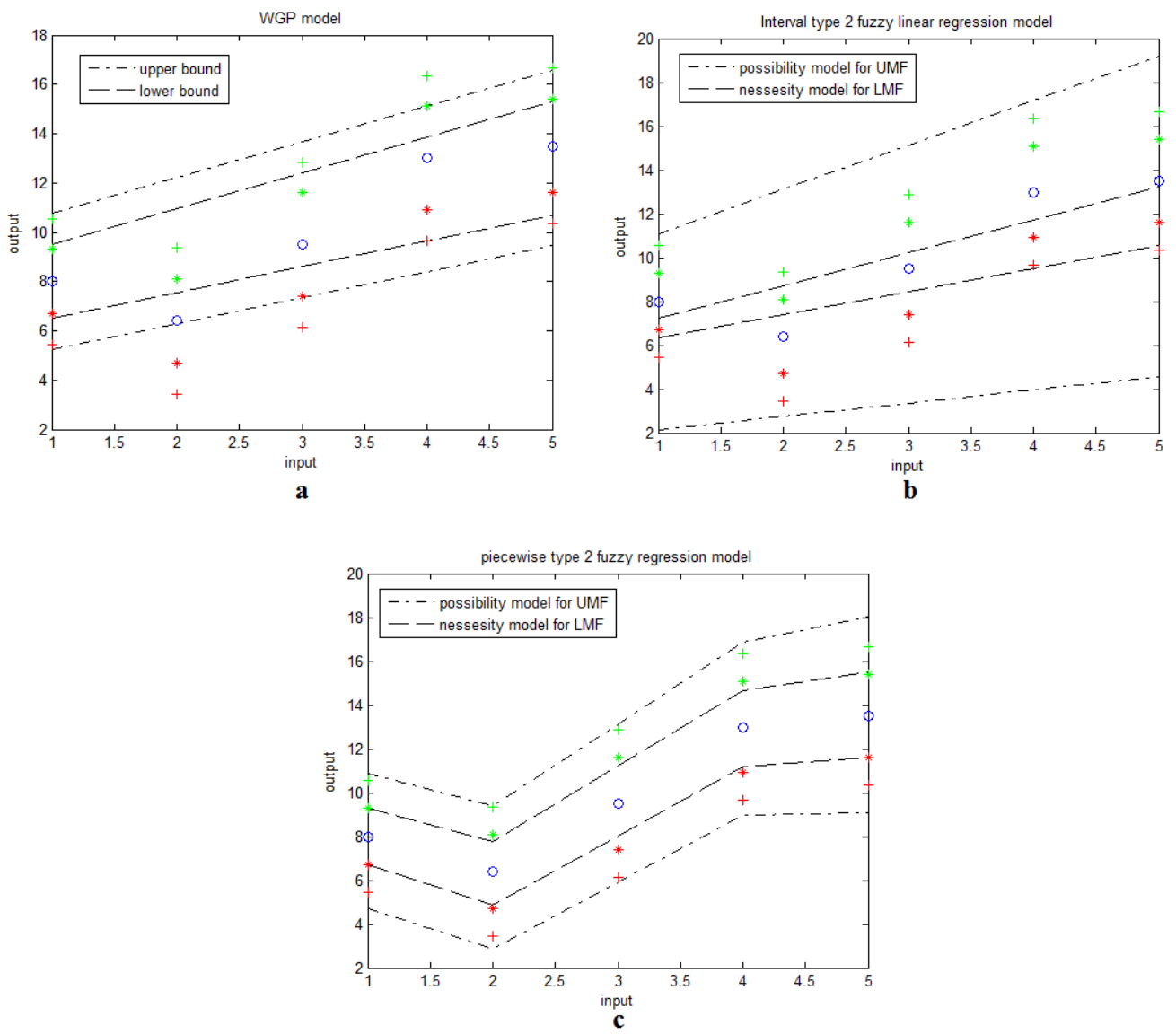

Fig. 4. a) WGP model ${ }^{17}$. b) Proposed interval T2FLR model. c) Proposed T2FLR model 


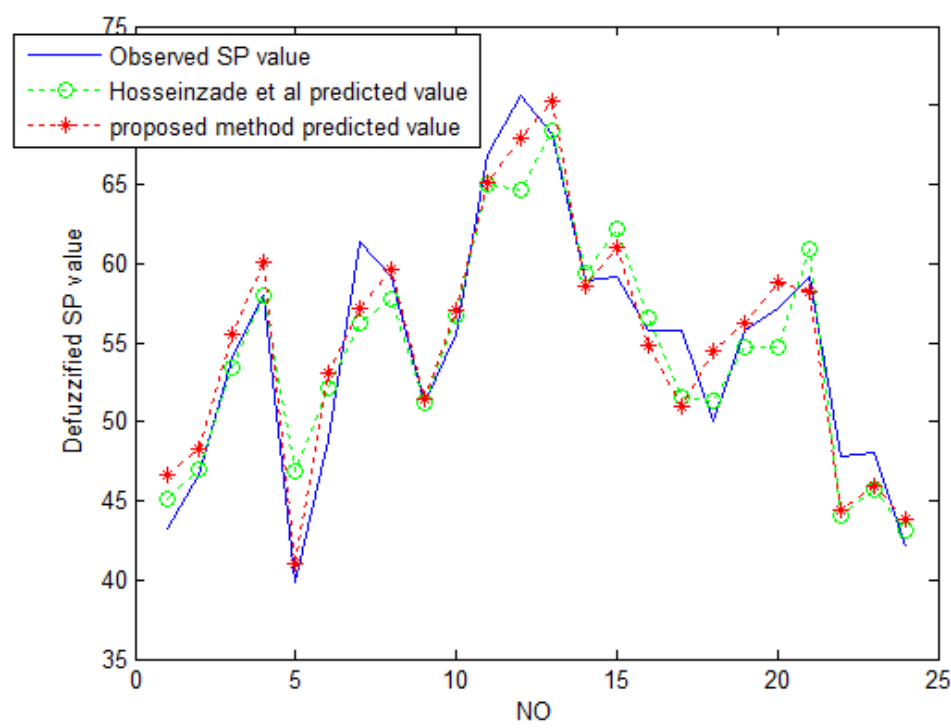

Fig. 5. Defuzzified Observed values and predicted values in multivariate T2FLR

In multivariate model, HS of our model is greater than WGP model, because HS has been defined based on the distances of supports of membership function like WGP model, but our model is based on the possibility and necessity models. This doesn't cause our research to be any less valuable. In fact, in most of the application, the results must be presented in a crisp form, which makes that RMSE comparison even more significant.

\section{Conclusions}

This study presents a type-2 fuzzy regression model according to the possibility and necessity model. In this model, vagueness is minimized, under the circumstances where the h-cut of observed value is included in predicted value. In this case, both observed values and predicted values are interval type- 2 fuzzy numbers. In this model both primary and secondary membership function of predicted value fit the observed value. Developing model to piecewise model makes it helpful in dealing with the fluctuating data.

The numerical examples in this study are more accurate than the ones presented in the existing literature. According to the numerical examples, the matching of the predicted and the observed values is observed to have happened closer than the ones reported in the previous works. In most application, results should be reported in the crisp form and in proposed model the results are more accurate after the defuzzification, which is shown by RMSE.
Only one type of type-2 fuzzy sets is discussed here, but many types of type-2 fuzzy sets can be treated through developing same concept as in this paper. Yet, most of high vague phenomenon might be well identified by this model.

\section{References}

1. Tanaka H, Uejima S, Asai K, Linear Regression Analysis with Fuzzy Model, IEEE Trans Syst MAN, Cybern. 75(6) (1982) 903-907.

2. Tanaka H, Fuzzy data analysis by possibilistic linear models, Fuzzy Sets Syst. 24(3) (1987) 363-375.

3. Tanaka H, Possibillistic linear systems and their application to the linear regression model, Fuzzy Sets Syst. 27(3) (1988) 275-289.

4. Phil Diamond, fuzzy least squares, Inf $\operatorname{Sci}(N y)$. 46(3) (1988) 141-157.

5. Lee HT, Chen SH, Fuzzy regression model with fuzzy input and output data for manpower forecasting, Fuzzy Sets Syst. 119(2) (2001) 205-213.

6. Huang C, Tzeng G, Multiple generation product life cycle predictions using a novel two-stage fuzzy piecewise regression analysis method, Technol Forecast Soc Change. 75(1) (2008) 12-31.

7. Pan N-F, Ko C-H, Yang M-D, Hsu K-C, Pavement performance prediction through fuzzy regression. Expert Syst Appl, 38(8) (2011) 10010-10017.

8. Kazemi A, Shakouri HG, Menhaj MB, Mehregan MR, Neshat N, A Hierarchical Fuzzy Linear Regression Model for Forecasting Agriculture Energy Demand: A Case Study of Iran, In Proc. 3rd Int. Conf. Information and Financial Engineering (Singapore, 2011), pp. 19-24. 
9. Choi $\mathrm{SH}$, Yoon JH, General fuzzy regression using least squares method, Int J Syst Sci. 41(5) (2010) 477-485.

10. Hojati M, Bector CR, Smimou K, A simple method for computation of fuzzy linear regression, Eur J Oper Res. 166(1) (2005) 172-184.

11. Özelkan EC, Duckstein L, Multi-objective fuzzy regression: a general framework, Comput Oper Res. 27(78) (2000) 635-652.

12. Peters G, Fuzzy linear regression with fuzzy intervals, Fuzzy Sets Syst. 119(2) (1994) 247-257.

13. Sentürka S, Fuzzy Regression Control Chart Based on $\alpha$ cut Approximation, Int J Comput Intell Syst. 3(1) (2010) 123-140.

14. Hamrawi H, Coupland S, John R, A novel alpha-cut representation for type-2 fuzzy sets, In Proc. 3rd Int. IEEE Conf. Fuzzy Systems (FUZZ) (Barcelona, 2010), pp. 1-8.

15. Wei Y, Watada J, Building a Type II Fuzzy Qualitative Regression, Intell Decis Technol. 1(15) (2012) 145-154.

16. Poleshchuk O, Komarov E, A fuzzy linear regression model for interval type-2 fuzzy sets, Annu Meet North Am Fuzzy Inf Process Soc. (2012), pp. 1-5.

17. Hosseinzadeh E, Hassanpour H, Arefi M, A weighted goal programming approach to fuzzy linear regression with crisp inputs and type-2 fuzzy outputs, Soft Comput. 19(5) (2014) 1143-1151.

18. Shafaei Bajestani N, Kamyad AV, Zare A, Possibilistic Type-2 Fuzzy Regression with Application to TAIEX Forecasting, Majlesi J Mechatron Syst. 5(2) (2016) 11-15.
19. Shafaei Bajestani N, Vahidian Kamyad A, Zare A, An Interval Type-2 Fuzzy Regression Model with Crisp Inputs and Type-2 Fuzzy Outputs for TAIEX Forecasting, In Proc. IEEE Conf. Information and Automation. (China 2016).

20. Tanaka H, Lee $H$, Interval Regression Analysis by Quadratic Programming Approach, IEEE Trans FUZZY Syst. 6(4) (1998) 473-481.

21. Hagras H, Wagner C, Towards the Wide Spread Use of Type-2 Fuzzy Logic Systems in Real World Applications, Comput Intell Mag IEEE. 7(3) (2012) 14-24.

22. Shafaei Bajestani N, Zare A, Forecasting TAIEX using improved type 2 fuzzy time series, Expert Syst Appl. 38(5) (2011) 5816-5821.

23. Betül Özkana, İhsan Kayaa, Ufuk Cebecib HB, A Hybrid Multicriteria Decision Making Methodology Based on Type-2 Fuzzy Sets For Selection Among Energy Storage Alternatives, Int J Comput Intell Syst. 8(5) (2015) 914927.

24. Sezi Cevik Onara, Başar Oztaysia CK, Strategic Decision Selection Using Hesitant fuzzy TOPSIS and Interval Type-2 Fuzzy AHP: A case study, Int J Comput Intell Syst. 7(5) (2014) 1002-1021.

25. J.Mendel, Uncertain Rule-Based Fuzzy Logic Systems: Introduction and New Directions (Prentice Hall, 2001).

26. Mencattini A, Salmeri M, Lojacono R, Type-2 Fuzzy Sets for Modeling Uncertainty in Measurement, In Int. Workshop on Advanced Methods for Uncertainty Estimation in Measurement. ( 2006), pp. 8-13. 